\title{
A LOWER BOUND FOR THE SPECTRUM OF THE LAPLACIAN IN TERMS OF SECTIONAL AND RICCI CURVATURE
}

\author{
ALBERTO G. SETTI
}

(Communicated by Barbara L. Keyfitz)

\begin{abstract}
Let $M$ be an $n$-dimensional, complete, simply connected Riemannian manifold. In this paper we show that if the sectional curvature is bounded above by $-k \leq 0$ and the Ricci curvature is bounded above by $-\alpha \leq 0$, then the spectrum of the Laplacian on $M$ is bounded below by $[\alpha+(n-1)(n-2) k] / 4$. This improves a previous result due to H. P. McKean.
\end{abstract}

\section{INTRODUCTION}

McKean [4] has shown that if $M$ is an $n$-dimensional complete, simply connected Riemannian manifold with sectional curvature satisfying $K \leq-k<$ 0 , then the bottom of the spectrum $\lambda_{0}$ of the Laplace operator $\Delta$, defined as a positive selfadjoint operator on $L^{2}(M)$ (cf. [5]), is bounded below by $(n-1)^{2} k / 4$.

While this bound is sharp, as shown by the case of the Laplacian on the hyperbolic space [1], [4], it is easy to see that even in very simple cases McKean's inequality fails to provide good bounds for $\lambda_{0}$.

Consider for instance a product manifold $M=M_{1} \times M_{2}$, where $M_{i}$ is a complete, $n_{i}$-dimensional, simply connected manifold with constant sectional curvature $-k_{i}<0$. Since the sectional curvature of the planes $\operatorname{span}\left\{X_{1}, X_{2}\right\} \subset$ $T M$, where $X_{i} \in T M_{i}$ vanishes, McKean's inequality does not give any bound for $\lambda_{0}$.

On the other hand, denoting by $\Delta_{i}$ the Laplacian on $M_{i}$ and by $\lambda_{0}^{(i)}$ the bottom of its spectrum, we have [1], [4] $\lambda_{0}^{(i)}=\left(n_{i}-1\right)^{2} k_{i} / 4$ and, since spectrum $(\Delta)$ $=\operatorname{spectrum}\left(\Delta_{1}\right)+\operatorname{spectrum}\left(\Delta_{2}\right)$, this implies $\lambda_{0}=\left(n_{1}-1\right)^{2} k_{1} / 4+\left(n_{2}-1\right)^{2} k_{2} / 4$.

The purpose of this article is to prove the following generalization of McKean's result:

Theorem 1. Let $M$ be an n-dimensional, complete, simply connected Riemannian manifold with sectional curvature $K$ and Ricci curvature Ric satisfying $K \leq-k \leq 0$ and $\operatorname{Ric} \leq-\alpha<0$. Then $\lambda_{0} \geq[\alpha+(n-1)(n-2) k] / 4$.

Received by the editors November 8, 1989 and, in revised form, March 5, 1990.

1980 Mathematics Subject Classification (1985 Revision). Primary 58G25; Secondary 35P15.

During the preparation of this work the author was supported by a Sloan Foundation Dissertation Fellowship. 
Observe that, if $K \leq-k<0$, then Ric $\leq-(n-1) k$, so $\lambda_{0} \geq(n-1)^{2} k / 4$. Thus Theorem 1 implies McKean's inequality. Moreover, if $M$ is a product manifold $M=M_{1} \times M_{2}$, then $\operatorname{Ric}^{M_{i}} \leq-\alpha_{i}$ implies $\operatorname{Ric}^{M} \leq-\min \left\{\alpha_{1}, \alpha_{2}\right\}$, where $\mathrm{Ric}^{M_{i}}, \mathrm{Ric}^{M}$ denote the Ricci curvature of $M_{i}$ and of $M$, respectively. In the example above, where $M_{i}$ is a space form of dimension $n_{i}$ and sectional curvature $-k_{i}<0$, Theorem 1 gives $\lambda_{0} \geq \min \left\{\left(n_{1}-1\right) k_{1},\left(n_{2}-1\right) k_{2}\right\} / 4$. This bound, even though not optimal, shows that Theorem 1 is an improvement of McKean's result.

\section{Notation}

For the proof of Theorem 1 we will follow Chavel's notation [1, pp. 65-67]: Given $v \in T_{p} M,|v|=1$, let $v^{\perp} \subseteq T_{p} M$ be the orthogonal complement of $v$, and let $\tau_{t}: T_{p} M \rightarrow T_{\exp t v} M$ be the parallel translation along the geodesic $\gamma_{v}(t)=\exp _{p} t v$. Denote by $\mathscr{A}(t, v): v^{\perp} \rightarrow v^{\perp}$ the linear transformation defined by

$$
\mathscr{A}(t, v) \eta=\left(\tau_{t}\right)^{-1} Y(t) \quad \forall \eta \in v^{\perp},
$$

where $Y(t)$ is the orthogonal Jacobi vector field along $\gamma_{v}$ determined by the initial conditions $Y(0)=0, \nabla_{t} Y(0)=\eta$. Thus [1, p. 63] $\mathscr{A}(t, v) \eta=$ $\tau_{t}^{-1}\left(T_{t v} \exp _{p}\right)(t \eta)$. Also let $\sqrt{g}(t, v)=\operatorname{det} \mathscr{A}(t, v)$. Using geodesic spherical coordinates at $p,(t, v) \in \mathbb{R}_{+} \times S T_{p} M$, we can write the metric in the form (cf. [1])

$$
d s^{2}=(d t)^{2}+|\mathscr{A}(t, v) d v|^{2}
$$

and the volume element of $M$ as

$$
d V(\exp t v)=\sqrt{g}(t, v) d t d \mu_{p}(v),
$$

where $d \mu_{p}$ denotes the $(n-1)$-dimensional measure on the unit sphere $S T_{p} M$ of $T_{p} M$.

For $\eta \in v^{\perp}$, also let $\mathscr{R}(t, v) \eta=\left(\tau_{t}\right)^{-1} R\left(\tau_{t} v, \tau_{t} \eta\right) \cdot \tau_{t} v$, where $R$ is the curvature tensor of $M$. Then (cf. $[1$, p. 66]) $\mathscr{R}(t, v)$ is a selfadjoint transformation of $v^{\perp}$ and $\mathscr{A}(t, v)$ satisfies the differential equation

$$
\mathscr{A}^{\prime \prime}(t, v)+\mathscr{R}(t, v) \mathscr{A}(t, v)=0
$$

with initial conditions $\mathscr{A}(0, v)=0, \mathscr{A}^{\prime}(0, v)=\mathrm{Id}_{v^{\perp}}$.

\section{THE MAIN GeOMETRIC ESTIMATE}

Theorem 2. Let $M$ be a complete, simply connected n-dimensional Riemannian manifold satisfying $K \leq-k \leq 0$ and $\mathrm{Ric} \leq-\alpha \leq 0$. Then

$$
\frac{1}{\sqrt{g}(t, v)} \frac{\partial}{\partial t} \sqrt{g}(t, v) \geq[\alpha+(n-1)(n-2) k]^{1 / 2} .
$$


The proof follows from the two lemmas below:

Lemma 3. With the same notations and hypotheses as in Theorem 2, we have

$$
\frac{1}{\sqrt{g}(t, r)} \frac{\partial^{2}}{\partial t^{2}} \sqrt{g}(t, v) \geq \alpha+(n-1)(n-2) k \text {. }
$$

Proof of Lemma 3. Following Chavel, let $U(t, v)=\mathscr{A}^{\prime}(t, v) \mathscr{A}^{-1}(t, v): v^{\perp} \rightarrow$ $v^{\perp}$. Observe that $\tau_{t} U(t, v) \tau_{t}^{-1}$ is the second fundamental form at $\exp _{p} t v$ of the geodesic sphere of radius $t$ at $p$.

It is easy to see (cf. [1, p. 72]) that $U(t, v)$ is a selfadjoint map of $v^{\perp}$ and that $U$ satisfies the matrix Riccati equation

$$
U^{\prime}+U^{2}+\mathscr{R}=0
$$

so that, taking the trace, we have

$$
(\operatorname{tr} U)^{\prime}+\operatorname{tr}\left(U^{2}\right)+\operatorname{tr} \mathscr{R}(t, v)=0 .
$$

Notice now that:

(i) $\operatorname{tr} U=\operatorname{tr}\left(\mathscr{A}^{\prime}(t, v) \mathscr{A}^{-1}(t, v)\right)=(\ln (\operatorname{det} \mathscr{A}))^{\prime}=\frac{1}{\sqrt{g}(t, v)} \frac{\partial}{\partial t} \sqrt{g}(t, v)$

(ii) $\operatorname{tr} \mathscr{R}(t, v)=\operatorname{Ric}\left(\dot{\gamma}_{v}(t), \dot{\gamma}_{v}(t)\right)$ where $\gamma_{v}(t)=\exp _{p} t v$.

Then we have

$$
\begin{aligned}
& \frac{1}{\sqrt{g}(t, v)} \frac{\partial^{2}}{\partial t^{2}} \sqrt{g}(t, v) \\
& \quad=\frac{\partial}{\partial t}\left\{\frac{1}{\sqrt{g}(t, v)} \frac{\partial}{\partial t} \sqrt{g}(t, v)\right\}+\left\{\frac{1}{\sqrt{g}(t, v)} \frac{\partial}{\partial t} \sqrt{g}(t, v)\right\}^{2} \\
& \quad=\frac{\partial}{\partial t}(\operatorname{tr} U)+(\operatorname{tr} U)^{2} \\
& \quad=\left[(\operatorname{tr} U)^{\prime}+\operatorname{tr}\left(U^{2}\right)\right]+\left[(\operatorname{tr} U)^{2}-\operatorname{tr}\left(U^{2}\right)\right] \\
& =-\operatorname{Ric}\left(\dot{\gamma}_{v}(t), \dot{\gamma}_{v}(t)\right)+(\operatorname{tr} U)^{2}-\operatorname{tr}\left(U^{2}\right) \\
& \geq \alpha+(\operatorname{tr} U)^{2}-\operatorname{tr}\left(U^{2}\right) .
\end{aligned}
$$

Since $U$ is selfadjoint, to complete the proof of the lemma it suffices to show that all the eigenvalues $\rho_{i}$ of $U$ are $\geq \sqrt{k}$, for then

$$
\begin{aligned}
(\operatorname{tr} U)^{2}-\operatorname{tr}\left(U^{2}\right) & =\left(\sum_{i=1}^{n-1} \rho_{j}\right)^{2}-\sum_{j=1}^{n-1} \rho_{j}^{2} \\
& =\sum_{j \neq i} \rho_{i} \rho_{j} \geq(n-1)(n-2) k .
\end{aligned}
$$

Therefore we need to show that

$$
\langle U(t, v) \eta, \eta\rangle /\langle\eta, \eta\rangle \geq \sqrt{k} \quad \forall \eta \in v^{\perp}, \forall t>0 .
$$

For $t_{0}$ fixed, let $\eta=\mathscr{A}\left(t_{0}, v\right) \zeta$, so that $\eta=\tau_{t}^{-1} Y\left(t_{0}\right)$, where $Y$ is the orthogonal Jacobi vector field along $\gamma_{v}(t)=\exp _{p} t v$ determined by the initial 
conditions $Y(0)=0, \nabla_{t} Y(0)=\zeta$. Then we have

$$
\begin{aligned}
\left\langle U\left(t_{0}\right) \eta, \eta\right\rangle & =\left\langle\mathscr{A}^{\prime}\left(t_{0}, v\right) \mathscr{A}\left(t_{0}, v\right)^{-1} \eta, \eta\right\rangle \\
& =\left\langle\mathscr{A}^{\prime}\left(t_{0}, v\right) \zeta, \mathscr{A}\left(t_{0}, v\right) \zeta\right\rangle \\
& =\left\langle\tau_{t_{0}} \mathscr{A}^{\prime}\left(t_{0}, v\right) \zeta, \tau_{t_{0}} \mathscr{A}\left(t_{0}, v\right) \zeta\right\rangle .
\end{aligned}
$$

By definition, $\tau_{t_{0}} \mathscr{A}\left(t_{0}, v\right) \zeta=Y\left(t_{0}\right)$, and it is easy to see that $\tau_{t_{0}} \mathscr{A}^{\prime}\left(t_{0}, v\right) \zeta=$ $\nabla_{t} Y\left(t_{0}\right)$, so that

$$
\left\langle U\left(t_{0}\right) \eta, \eta\right\rangle=\left\langle\nabla_{t} Y\left(t_{0}\right), Y\left(t_{0}\right)\right\rangle=|Y|\left(t_{0}\right)|Y|^{\prime}\left(t_{0}\right) .
$$

Since the sectional curvature of $M$ is bounded above by $-k$, we obtain, applying Rauch's Comparison Theorem (cf. [1, p. 67])

$$
|Y|^{\prime}\left(t_{0}\right) /|Y|\left(t_{0}\right) \geq \psi^{\prime}\left(t_{0}\right) / \psi\left(t_{0}\right),
$$

where

$$
\begin{aligned}
\psi(t) & =|Y(0)| \cosh (\sqrt{k} t)+|Y|^{\prime}(0) \frac{1}{\sqrt{k}} \sinh (\sqrt{k} t) \\
& =|Y|^{\prime}(0) \frac{1}{\sqrt{k}} \sinh (\sqrt{k} t) .
\end{aligned}
$$

Hence

$$
|Y|^{\prime}\left(t_{0}\right) /|Y|\left(t_{0}\right) \geq \sqrt{k} \operatorname{coth}\left(\sqrt{k} t_{0}\right) \geq \sqrt{k}
$$

and, substituting in (3),

$$
\left\langle U\left(t_{0}\right) \eta, \eta\right\rangle /\langle\eta, \eta\rangle \geq \sqrt{k},
$$

therefore completing the proof of Lemma 3.

Lemma 4. Let $M$ be a complete, simply connected Riemannian manifold with nonpositive sectional curvature. Assume that $\frac{1}{\sqrt{g}(t, v)} \frac{\partial^{2}}{\partial t^{2}} \sqrt{g}(t, v) \geq \beta>0$. Then

$$
\frac{1}{\sqrt{g}(t, v)} \frac{\partial}{\partial t} \sqrt{g}(t, v) \geq \sqrt{\beta} \text {. }
$$

Proof of Lemma 4. Notice that, by Bishop Comparison Theorem I ([1, pp. 6869]) the hypothesis on the sectional curvature implies that $\frac{1}{\sqrt{\bar{g}(t, v)}} \frac{\partial}{\partial t} \sqrt{g}(t, v) \geq$ 0 . Moreover, since $\mathscr{A}(t, v) \eta=\left(\tau_{t}\right)^{-1}\left(T_{t v} \exp _{p}\right)(t \eta)$, and $\left(\tau_{t}\right)^{-1}\left(T_{t v} \exp _{p}\right) \rightarrow$ $\mathrm{Id}_{v^{\perp}}$ as $t \downarrow 0$, we have $\sqrt{g}(t, v)=\operatorname{det} \mathscr{A}(t, v) \sim t^{n-1}$ as $t \downarrow 0$, so that $\frac{1}{\sqrt{g}(t, v)} \frac{\partial}{\partial t} \sqrt{g}(t, v) \sim \frac{n-1}{t}$ as $t \downarrow 0$ and $\frac{\partial}{\partial t}\left(\frac{1}{\sqrt{g}} \frac{\partial}{\partial t} \sqrt{g}\right)<0$ for $t$ small enough.

Then we write

$$
\frac{1}{\sqrt{g}} \frac{\partial^{2}}{\partial t^{2}} \sqrt{g}=\frac{\partial}{\partial t}\left(\frac{1}{\sqrt{g}} \frac{\partial}{\partial t} \sqrt{g}\right)+\left(\frac{1}{\sqrt{g}} \frac{\partial}{\partial t} \sqrt{g}\right)^{2} .
$$

Given $t_{0}$, if $\frac{\partial}{\partial t}\left(\frac{1}{\sqrt{g}} \frac{\partial}{\partial t} \sqrt{g}\right)\left(t_{0}\right) \leq 0$, then $\left(\frac{1}{\sqrt{g}} \frac{\partial}{\partial t} \sqrt{g}\right)^{2} \geq \frac{1}{\sqrt{g}} \frac{\partial^{2}}{\partial t^{2}} \sqrt{g} \geq \beta$ at $t_{0}$, and (4) holds at $t_{0}$. 
If $\frac{\partial}{\partial t}\left(\frac{1}{\sqrt{q}} \frac{\partial}{\partial t} \sqrt{g}\right)>0$ at $t_{0}$ let $t_{1} \leq\left(0, t_{0}\right)$ be the point where $\frac{1}{\sqrt{g}} \frac{\partial}{\partial t} \sqrt{g}$ has its minimum in $\left(0, t_{0}\right]$. Then $\frac{\partial}{\partial t}\left(\frac{1}{\sqrt{g}} \frac{\partial}{\partial t} \sqrt{g}\right)=0$ at $t_{1}$ and

$$
\left(\frac{1}{\sqrt{g}} \frac{\partial}{\partial t} \sqrt{g}\right)\left(t_{0}\right) \geq\left(\frac{1}{\sqrt{g}} \frac{\partial}{\partial t} \sqrt{g}\right)\left(t_{1}\right)=\left(\frac{1}{\sqrt{g}} \frac{\partial^{2}}{\partial t^{2}} \sqrt{g}\left(t_{1}\right)\right)^{1 / 2} \geq \sqrt{\beta} .
$$

Thus (4) holds for every $t>0$, and the proof of Lemma 4 is complete.

\section{Proof of Theorem 1}

The proof of Theorem 1 now follows familiar lines (cf. [1, p. 47], [5, pp. 67-69]). By Rayleigh's Theorem, it suffices to show that, $\forall f \in C_{c}^{\infty}(M)$,

$$
\int_{M}|\nabla f|^{2} d V \geq[\alpha+(n-1)(n-2)] / 4 \int_{M} f^{2} d V
$$

For convenience, set $\alpha+(n-1)(n-2)=\beta$. Using spherical geodesic coordinates $(t, v) \in \mathbb{R}_{+} \times S T_{p} M$ and, denoting the local expression of $f \in C_{c}^{\infty}(M)$ with the same symbol, we have, $\forall v \in S T_{p} M$,

$$
\begin{aligned}
\int_{0}^{\infty} f^{2}(t, v) \sqrt{g}(t, v) d t \leq & 1 / \sqrt{\beta} \int_{0}^{\infty} f^{2}(t, v) \frac{\partial}{\partial t} \sqrt{g}(t, v) d t \\
= & -2 / \sqrt{\beta} \int_{0}^{\infty} f(t, v) \frac{\partial f}{\partial t}(t, v) \sqrt{g}(t, v) d t \\
\leq & \frac{2}{\sqrt{\beta}}\left(\int_{0}^{\infty} f^{2}(t, v) \sqrt{g}(t, v) d t\right)^{1 / 2} \\
& \times\left(\int_{0}^{\infty}\left[\frac{\partial f}{\partial t}(t, v)\right]^{2} \sqrt{g}(t, v) d t\right)^{1 / 2},
\end{aligned}
$$

where the first inequality follows from $\frac{\partial}{\partial t} \sqrt{g}(t, v) \geq \sqrt{\beta} \sqrt{g}(t, v)$ (Theorem 2 ), the equality on the second line is obtained by integrating by parts, and the last inequality is the Cauchy-Schwartz inequality.

Using the fact that $|\nabla f|^{2}(t, v) \geq\left[\frac{\partial}{\partial t} f(t, v)\right]^{2}$ and simplifying we find that

$$
\int_{0}^{\infty} f^{2}(t, v) \sqrt{g}(t, v) d t \leq 4 / \beta \int_{0}^{\infty}|\nabla f|^{2}(t, v) \sqrt{g}(t, v) d t
$$

whence, integrating over $v \in s^{n-1}$, we obtain

$$
\begin{aligned}
\int_{M} f^{2} d V & =\int_{S^{n-1}} \int_{0}^{\infty} f^{2}(t, v) \sqrt{g}(t, v) d t d \mu_{p}(v) \\
& \leq \frac{4}{\beta} \int_{S^{n-1}} \int_{0}^{\infty}|\nabla f|^{2}(t, v) \sqrt{q}(t, v) d t d \mu_{p}(v) \\
& \leq \frac{4}{\beta} \int_{M}|\nabla f|^{2} d V .
\end{aligned}
$$

This completes the proof of Theorem 1 . 
Remarks. As shown in [5], a lower bound for $\frac{1}{\sqrt{g}} \frac{\partial}{\partial t} \sqrt{g}$ also provides lower bounds for $\|\nabla f\|_{p} /\|f\|_{p}$ for every $p, 1 \leq p<\infty$, and $f$ compactly supported with $f, \nabla f \in L^{p}(M)$. Thus, in the hypotheses of Theorem 1 we have

$$
\|f\|_{p} \leq \frac{p}{[\alpha+(n-1)(n-2) k]^{1 / 2}}\|\nabla f\|_{p} .
$$

A lower bound to $\frac{1}{\sqrt{g}} \frac{\partial}{\partial t} \sqrt{g}$ can also be used to estimate the Cheeger's isoperimetric constant $h(M)$ of the manifold $M$ (cf. [1], [2], [6]). An argument as in [1, pp. 95-96], shows that $\frac{1}{\sqrt{g}} \frac{\partial}{\partial t} \sqrt{g} \geq[\alpha+(n-1)(n-2) k]^{1 / 2}$ implies $h(M) \geq[\alpha+(n-1)(n-2) k]^{1 / 2}$. This together with Cheeger's Theorem yields another proof of Theorem 1 .

\section{ACKNOWLEDGMENT}

I would like to thank my thesis advisor, Professor Robert S. Strichartz, for bringing this problem to my attention and for many stimulating and helpful discussions. I am also grateful to him for supplying the proof of Lemma 4.

\section{REFERENCES}

1. J. Chavel, Eigenvalues in Riemannian geometry, Academic Press, Orlando, 1984.

2. J. Cheeger, A lower bound for the smallest eigenvalue of the Laplacian in Problems in analysis, a symposium in honor of S. Bochner, Princeton University Press, Princeton, 1970, 195-199.

3. S. Y. Cheng, Eigenfunctions and eigenvalues of Laplacian, Proc. Symp. Pure Math. 27 (1975), 185-193.

4. H. P. McKean, An upper bound to the spectrum of $\Delta$ on a manifold of negative curvature, J. Differential Geometry 4 (1970), 359-366.

5. R. S. Strichartz, Analysis of the Laplacian on the complete Riemannian manifold, J. Funct. Anal. 52 (1983), 48-79.

6. S. T. Yau, Isoperimetric constants and the first eigenvalue of a compact Riemannian manifold, Ann. Sci. École Norm. Sup. (4) 8 (1975), 487-507.

Department of Mathematics, White Hall, Cornell University, Ithaca, New York 14853 